
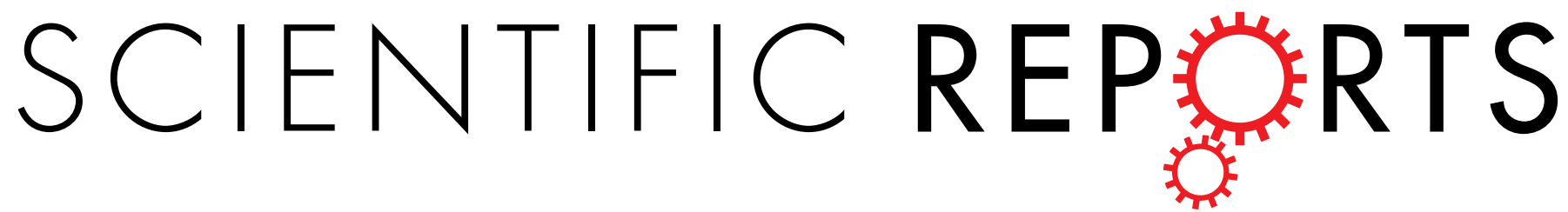

\title{
OPEN Voxel-based analysis of grey and white matter degeneration in cervical spondylotic myelopathy
}

Received: 02 October 2015

Accepted: 04 April 2016

Published: 20 April 2016

\author{
Patrick Grabher ${ }^{1}$, Siawoosh Mohammadi ${ }^{2,3}$, Aaron Trachsler $^{1}$, Susanne Friedl ${ }^{1}$, \\ Gergely David ${ }^{1}$, Reto Sutter ${ }^{4}$, Nikolaus Weiskopf ${ }^{3,5}$, Alan J. Thompson ${ }^{6}$, Armin Curt $^{1}$ \& \\ Patrick Freund ${ }^{1,3,5,6}$
}

In this prospective study, we made an unbiased voxel-based analysis to investigate above-stenosis spinal degeneration and its relation to impairment in patients with cervical spondylotic myelopathy (CSM). Twenty patients and 18 controls were assessed with high-resolution MRI protocols above the level of stenosis. Cross-sectional areas of grey matter (GM), white matter (WM), and posterior columns (PC) were measured to determine atrophy. Diffusion indices assessed tract-specific integrity of PC and lateral corticospinal tracts (CST). Regression analysis was used to reveal relationships between MRI measures and clinical impairment. Patients showed mainly sensory impairment. Atrophy was prominent within the cervical WM (13.9\%, $\mathrm{p}=0.004), \mathrm{GM}(7.2 \%, \mathrm{p}=0.043)$, and $\mathrm{PC}(16.1 \%, \mathrm{p}=0.005)$. Fractional anisotropy (FA) was reduced in the PC $(-11.98 \%, \mathrm{p}=0.006)$ and lateral CST $(-12.96 \%$, $\mathrm{p}=0.014)$. In addition, radial $(+28.47 \%, p=0.014)$, axial $(+14.72 \%, p=0.005)$, and mean $(+16.50 \%$, $\mathrm{p}=0.001$ ) diffusivities were increased in the $\mathrm{PC}$. Light-touch score was associated with atrophy $\left(R^{2}=0.3559, p=0.020\right)$ and FA ( $z$ score $\left.3.74, p=0.003\right)$ in the $P C$, as was functional independence and FA in the lateral CST ( $z$ score $3.68, p=0.020$ ). This study demonstrates voxel-based degeneration far above the stenosis at a level not directly affected by the compression and provides unbiased readouts of tract-specific changes that relate to impairment.

Cervical spondylotic myelopathy (CSM) is one of the most frequent spinal cord disorders leading to reduced independence and quality of life especially in the elderly ${ }^{1}$. Neurological impairment is mainly caused through chronic cord compression and deterioration of blood supply triggering degeneration of neural tissue in grey and white matter in the spinal cord at the level of stenosis ${ }^{2,3}$. The time course of the stenosis and its relation to the emergence of clinical impairment is unknown, as a moderate cord stenosis might be clinically silent for many years. Decompressive surgery is the therapeutic gold standard in patients with clinically relevant cord stenosis ${ }^{4}$, while a conservative approach is chosen in those patients with a radiologically less severe stenosis and only mild symptoms. Recently, novel experimental neuroprotective approaches improving outcome were investigated by blocking Fas-mediated apoptosis ${ }^{2}$ and the application of a benzothiazole anticonvulsant (i.e. Riluzole) ${ }^{5,6}$ and results were encouraging. Hence, there is a pressing need to detect and monitor early subclinical pathologic changes to the microstructure of the spinal cord, allowing interventions before those changes cause clinical impairments. However, neuroimaging biomarkers that can quantify disease progression at a stage before major irreversible damage occurs, monitor treatment effects (beyond decompression of the cord) and predict outcome are lacking ${ }^{1}$.

Recent developments in imaging and post-processing techniques have significantly advanced the accuracy and sensitivity of spinal cord neuroimaging. For example the advent of a common anatomical spinal cord template $^{7}$, probabilistic tracts ${ }^{7}$, and optimization of post-processing techniques ${ }^{8}$ have now opened the avenue for unbiased voxel-based morphometry (VBM) analysis (i.e. without an a priori hypothesis) of volumetric as well

${ }^{1}$ Spinal Cord Injury Center Balgrist, University Hospital Zurich, University of Zurich, Zurich, Switzerland. ${ }^{2}$ Department of Systems Neuroscience, University Medical Center Hamburg-Eppendorf, Hamburg, Germany. ${ }^{3}$ Wellcome Trust Centre for Neuroimaging, UCL Institute of Neurology, University College London, London, United Kingdom. ${ }^{4}$ Department of Radiology, University Hospital Balgrist, Zurich, Switzerland. ${ }^{5}$ Department of Neurophysics, Max Planck Institute for Human Cognitive and Brain Sciences, Leipzig, Germany. ${ }^{6}$ Department of Brain Repair and Rehabilitation, UCL Institute of Neurology, University College London, London, United Kingdom. Correspondence and requests for materials should be addressed to P.F. (email: pfreund@paralab.balgrist.ch) 


\begin{tabular}{|c|c|c|c|c|c|c|c|c|c|c|c|c|c|c|c|}
\hline & Gender & Age & $\begin{array}{l}\text { Level of } \\
\text { stenosis }\end{array}$ & mJOA & UEMS & UELT & UEPP & SCIM & $\begin{array}{l}\text { GRASSP } \\
\text { Strength }\end{array}$ & $\begin{array}{l}\text { GRASSP } \\
\text { Sensibility }\end{array}$ & $\begin{array}{l}\text { GRASSP } \\
\text { Prehension }\end{array}$ & $\begin{array}{c}\text { MSCC } \\
(\%)\end{array}$ & $\begin{array}{c}\text { MCC } \\
(\%)\end{array}$ & $\begin{array}{c}\mathrm{T} 2 \\
\text { Hyperintensity }\end{array}$ & $\begin{array}{c}\text { Signal } \\
\text { Change } \\
\text { Ratio }\end{array}$ \\
\hline 1 & $\mathrm{~m}$ & 36 & $\mathrm{C} 5 / 6^{\mathrm{a}}$ & 12 & 48 & 20 & 25 & 99 & 74 & 37 & 84 & 8.35 & 39.14 & No & 0.93 \\
\hline 2 & $\mathrm{~m}$ & 50 & $\mathrm{C} 5 / 6^{\mathrm{a}}$ & 16 & 49 & 25 & 26 & 100 & 100 & 47 & 84 & 23.85 & 48.37 & No & 1.03 \\
\hline 3 & $\mathrm{~m}$ & 39 & $\mathrm{C} 3 / 4^{\mathrm{a}}$ & 13 & 50 & 25 & 23 & 100 & 98 & 43 & 84 & 24.38 & 40.83 & No & 1.00 \\
\hline 4 & $\mathrm{f}$ & 66 & $\mathrm{C} 5 / 6$ & 12 & 49 & 32 & 32 & 96 & 95 & 37 & 84 & 14.14 & 36.67 & Yes & 1.33 \\
\hline 5 & $\mathrm{~m}$ & 69 & $\mathrm{C} 5 / 6^{\mathrm{a}}$ & 17 & 50 & 32 & 32 & 100 & 99 & 44 & 84 & 16.31 & 39.59 & No & 1.12 \\
\hline 6 & $\mathrm{~m}$ & 68 & $\mathrm{C} 6 / 7^{\mathrm{a}}$ & 17 & 50 & 32 & 32 & 100 & NT & NT & NT & 26.12 & 49.81 & Yes & 1.77 \\
\hline 7 & $\mathrm{~m}$ & 39 & $\mathrm{C} 5 / 6$ & 16 & 50 & 32 & 25 & 100 & 100 & 46 & 84 & 29.33 & 52.72 & No & 1.13 \\
\hline 8 & $\mathrm{~m}$ & 34 & $\mathrm{C} 5 / 6$ & 14 & 50 & 30 & 30 & 100 & 100 & 47 & 84 & 23.73 & 37.29 & No & 0.99 \\
\hline 9 & $\mathrm{f}$ & 31 & $\mathrm{C} 5 / 6$ & 16 & 50 & 27 & 27 & 100 & 100 & 48 & 84 & 11.40 & 34.04 & Yes & 1.55 \\
\hline 10 & $\mathrm{f}$ & 47 & $\mathrm{C} 5 / 6^{\mathrm{a}}$ & 16 & 50 & 31 & 31 & 100 & 100 & 48 & 84 & 12.84 & 39.94 & Yes & 1.35 \\
\hline 11 & $\mathrm{~m}$ & 63 & $\mathrm{C} 4 / 5^{\mathrm{a}}$ & 12 & 50 & 24 & 24 & 95 & 94 & 41 & 84 & 13.82 & 30.67 & Yes & 1.81 \\
\hline 12 & $\mathrm{~m}$ & 74 & $\mathrm{C} 6 / 7^{\mathrm{a}}$ & 14 & 50 & 32 & 32 & 88 & 89 & 42 & 84 & 22.62 & 43.01 & Yes & 1.34 \\
\hline 13 & $\mathrm{~m}$ & 32 & $\mathrm{C} 5 / 6^{\mathrm{a}}$ & 16 & 50 & 20 & 20 & 100 & 100 & 48 & 84 & 1.13 & 27.14 & None & 1.03 \\
\hline 14 & $\mathrm{~m}$ & 66 & $\mathrm{C} 5 / 6$ & 9 & 50 & 25 & 26 & 86 & 99 & 34 & 82 & 18.17 & 26.44 & Yes & 2.27 \\
\hline 15 & $\mathrm{~m}$ & 51 & C5/6 & 15 & 50 & 27 & 27 & 96 & 92 & 44 & 81 & 17.07 & 36.32 & No & 1.00 \\
\hline 16 & $\mathrm{f}$ & 53 & $\mathrm{C} 5 / 6$ & 16 & 50 & 32 & 30 & 100 & 100 & 46 & 84 & 18.76 & 49.98 & Yes & 1.46 \\
\hline 17 & $\mathrm{~m}$ & 72 & $\mathrm{C} 7 / \mathrm{T} 1^{\mathrm{a}}$ & 14 & 50 & 26 & 22 & 100 & 98 & 41 & 83 & 25.82 & 32.07 & No & 1.05 \\
\hline 18 & $\mathrm{f}$ & 37 & $\mathrm{C} 3 / 4^{\mathrm{a}}$ & 14 & 49 & 30 & 30 & 99 & 93 & 46 & 79 & 16.03 & 23.00 & Yes & 1.31 \\
\hline 19 & $\mathrm{f}$ & 58 & $\mathrm{C} 5 / 6^{\mathrm{a}}$ & 16 & 49 & 29 & 29 & 100 & 93 & 48 & 84 & 15.64 & 34.96 & No & 1.00 \\
\hline 20 & $\mathrm{~m}$ & 55 & C6/7 & 12 & 50 & 23 & 23 & 98 & 84 & 22 & 81 & 7.15 & 25.25 & No & 1.06 \\
\hline
\end{tabular}

Table 1. Clinical and behavioural data of 20 patients with cervical spondylotic myelopathy. $\mathrm{mJOA}=$ modified Japanese Orthopaedic Association [max. 18 points]. UEMS $=$ Upper extremity motor score [max. 50 points]. UELT $=$ Upper extremity light-touch [max. 32 points]. UEPP $=$ Upper extremity pinprick [max. 32 points]. SCIM = Spinal Cord Independence Measure [max. 100 points]. GRASSP $=$ Graded Redefined Assessment of Strength, Sensibility and Prehension [strength: max. 100 points, sensibility: max. 48 points, prehension: $\max .84$ points]. MSCC = Maximum Spinal Cord Compression. MCC = Maximum Canal Compromise. NT $=$ Not tested. ${ }^{a}$ Multisegmental degeneration of cervical spine.

as microstructural changes ${ }^{7,8}$. This enables the spatial localization of cord pathology and the determination of its relation with clinical impairment ${ }^{9,10}$.

In this study, we took advantage of high-resolution anatomical volumetric MRI to assess grey and white matter atrophy (i.e. Multiple Echo Data Image Combination (MEDIC)) and DTI to assess changes to the microstructural tissue properties. We hypothesised that chronic cord compression results in remote and spatially localized morphometric changes (i.e. atrophy) within cervical grey and white matter as well as tract-specific changes above the level of stenosis in CSM patients and that the degree of remote degeneration relates to clinical impairment.

\section{Results}

Subjects. In patients, impairment was assessed on the modified Japanese Orthopaedic Association (mJOA) score which identified ten patients suffering from mild (mJOA $\geq 15$ [max. 18]), nine from moderate $(\mathrm{mJOA}=12-14)$ and one from severe $(\mathrm{mJOA}<12)$ CSM. The upper-extremity International Standards for Neurological Classification of SCI (ISNCSCI) scores were (mean \pm SD) $27.70 \pm 4.07$ for light-touch (UELT) [max. 32], 27.30 \pm 3.77 for pin-prick (UEPP) [max. 32], and 49.70 \pm 0.57 for motor score (UEMS) [max. 50]. The spinal cord independence measure (SCIM) was 97.85 \pm 4.04 [max. 100]. The total Graded Redefined Assessment of Strength, Sensibility and Prehension (GRASSP) score was 220.74 \pm 12.32 [max. 232], and the sub-items were $95.16 \pm 6.83$ for strength [max. 100], $42.57 \pm 6.50$ for sensibility [max. 48], and $83.26 \pm 1.45$ for prehension [max. 84]. Individual scores are shown in Table 1.

Conventional MRI measures at site of stenosis. The level of stenosis was at $\mathrm{C} 5 / \mathrm{C} 6(\mathrm{n}=13)$ for the majority of patients, twice at $\mathrm{C} 3 / 4$, once at $\mathrm{C} 4 / \mathrm{C} 5$, three times at $\mathrm{C} 6 / 7$, and once at $\mathrm{C} 7 / \mathrm{C} 8$. In patients, the maximum spinal cord compression (MSCC) was $17.33 \pm 7.21 \%$ and maximum canal compromise (MCC) $37.36 \pm 8.58 \%$. Nine patients showed a T2 signal hyperintensity with a signal change ratio of $1.58 \pm 0.32$. Individual data are shown in Table 1.

Morphometric changes in the cervical cord above the site of stenosis. Volumetric as well as microstructural changes were observed in patients compared to controls above the level of stenosis at C2/C3. In patients, the cross-sectional cervical cord area was reduced by $12.8 \%$ (patients: $80.88 \mathrm{~mm}^{2}$, CI $75.59-86.17 \mathrm{~mm}^{2}$ vs. controls: $92.77 \mathrm{~mm}^{2}$, CI $88.88-96.67 \mathrm{~mm}^{2}, \mathrm{p}=0.0032$ ), GM area by $7.2 \%$ (patients: $17.80 \mathrm{~mm}^{2}$, CI $16.96-$ $18.64 \mathrm{~mm}^{2}$ vs. controls: $19.18 \mathrm{~mm}^{2}$, CI $18.33-20.03 \mathrm{~mm}^{2}, \mathrm{p}=0.0429$ ), WM area by $13.9 \%$ (patients: $63.08 \mathrm{~mm}^{2}$, CI $58.42-67.75 \mathrm{~mm}^{2}$ vs. controls: $73.23 \mathrm{~mm}^{2}$, CI $69.88-76.57 \mathrm{~mm}^{2}, \mathrm{p}=0.0041$ ) and PC area by $16.1 \%$ (patients: $18.86 \mathrm{~mm}^{2}$, CI $17.30-20.41 \mathrm{~mm}^{2}$ vs. controls: $22.49 \mathrm{~mm}^{2}$, CI $21.06-23.92 \mathrm{~mm}^{2}, \mathrm{p}=0.0051$ ) (Fig. 1). 


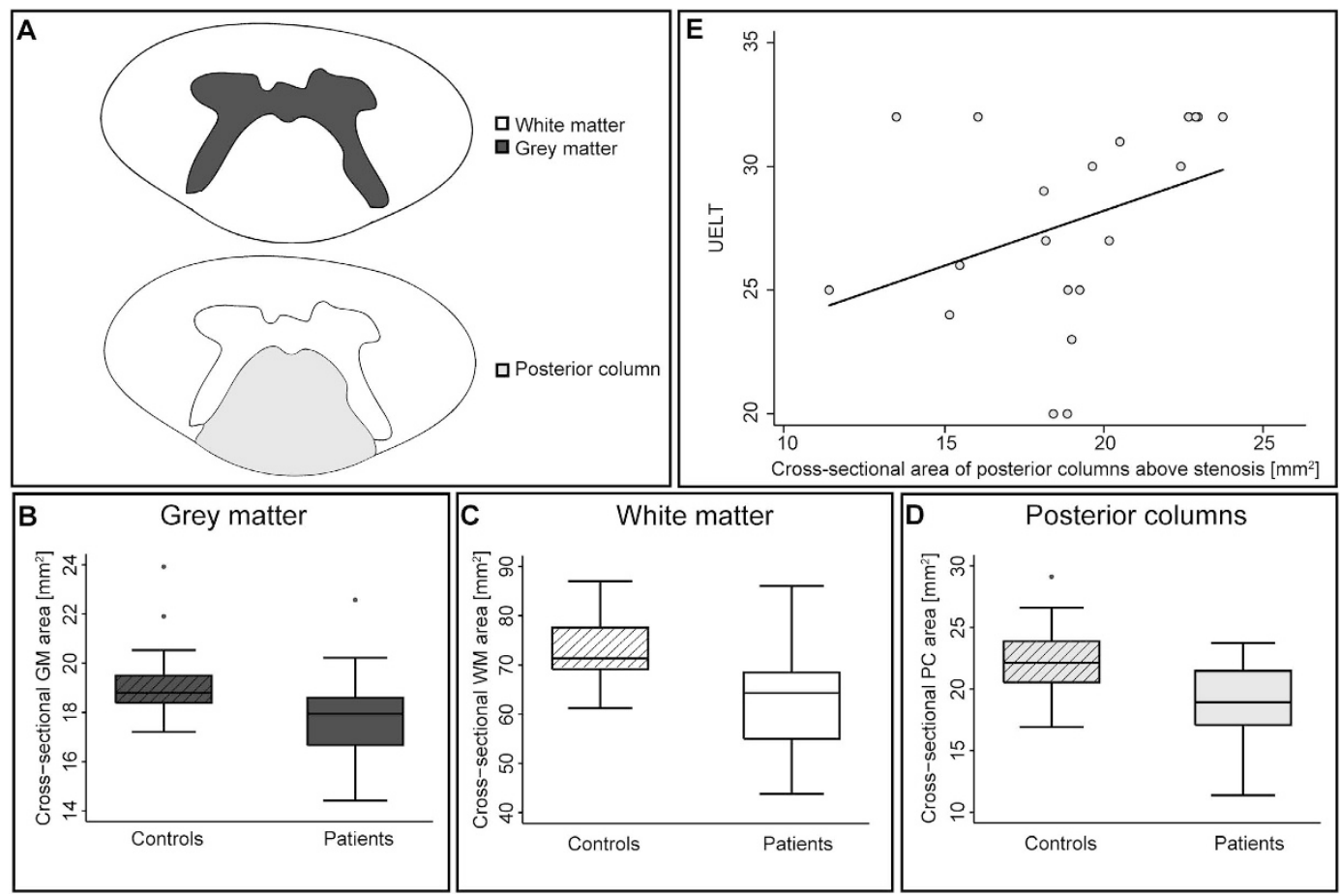

Figure 1. Morphometric changes in cervical spinal cord above compression site at C2/C3. (A) Schematic illustration of segmented cross-sectional cervical areas of grey matter, white matter, and posterior columns remote to compression. Significant reductions are shown for cross-sectional cervical cord area of grey matter (B), white matter $(\mathbf{C})$, and posterior columns (D) in patients compared to controls. (E) Correlation between ISNCSCI upper extremity light-touch (UELT) score and cross-sectional area of the posterior columns (for illustrative purposes, not adjusted for age).

Voxel-based analysis of the cervical cord DTI revealed reductions in fractional anisotropy (FA) in the lateral CST ( $-12.96 \%$, patients: 0.57 , CI $0.53-0.61$ vs. controls: 0.66 , CI 0.63-0.68; x:4.5, y: -20.5 , z:8.0, z score $=3.30$, cluster extent $=144, \mathrm{p}=0.014$, FWE corrected $)$ and PC $(-11.98 \%$, patients: 0.59 , CI $0.55-0.63$ vs. controls: 0.67 , CI 0.65-0.69; $\mathrm{x}:-2.0, \mathrm{y}:-20.0, \mathrm{z}: 8.0$, z score $=4.08$, cluster extent $=174, \mathrm{p}=0.006$, FWE corrected) while axial diffusivity $(\mathrm{AD})(+14.72 \%$, patients: 0.0019 , CI $0.0019-0.0020$ vs. controls: 0.0017 , CI $0.0016-0.0017$; $\mathrm{x}:-1.0, \mathrm{y}:-18.5, \mathrm{z}: 41.0, \mathrm{z}$ score $=3.91$, cluster extent $=228, \mathrm{p}=0.005$, FWE corrected), radial diffusivity (RD) (+28.47\%, patients: 0.00073 , CI 0.00064-0.00082 vs. controls: 0.00057, CI 0.00053-0.00060; x:1.0, y:-18.0, z:14.0, $\mathrm{z}$ score $=3.39$, cluster extent $=203, \mathrm{p}=0.014$, FWE corrected $)$, and mean diffusivity $(\mathrm{MD})(+16.50 \%$, patients: 0.00073 , CI $0.00064-0.00082$ vs. controls: 0.00057 , CI $0.00053-0.00060 ; \mathrm{x}: 2.0, \mathrm{y}:-19.5, \mathrm{z}: 41.0$, z score $=3.80$, cluster extent $=379, \mathrm{p}=0.001$, FWE corrected) were all increased in the PCs in patients when compared to controls (Figs 2 and 3).

Clinical associations. In patients, significant associations were seen between macro- and microstructural MRI readouts and impairment. In particular, reduced PC area was associated with lower ISNCSCI UELT scores $\left(\mathrm{R}^{2}=0.3559, \mathrm{p}=0.020\right)$ (Fig. 1E). Furthermore, lower FA in the left PC was associated with lower left ISNCSCI UELT scores (x: 0.0, y: $-18.5, \mathrm{z}: 19.0$, $\mathrm{z}$ score $=3.74, \mathrm{p}=0.003$, extent $=198$, FWE corrected) (Fig. 4A). Lower FA in the left lateral CST was associated with lower SCIM score $(\mathrm{x}:-3.5, \mathrm{y}:-21.5, \mathrm{z}:-3.0, \mathrm{z}$ score $=3.68, \mathrm{p}=0.020$, extent $=135$, FWE corrected) (Fig. 4B).

\section{Discussion}

This study in patients with CSM shows, next to conventional MRI measures (e.g. MSCC, MCC, signal change ratio $)^{11}$, for the first time remote and spatially localized morphological and microstructural changes of the spinal cord within the grey and white matter above the level of stenosis. In particular, there was grey and white matter atrophy, which was paralleled by tract-specific microstructural changes in major spinal pathways. Crucially, the degree of spinal cord pathology correlated with measures of clinical impairment of posterior column and pyramidal dysfunction. Thus, unbiased voxel-based analysis revealing spatially localized cord pathology provides complementary pathophysiological insights into rostral degenerative changes beyond the routine clinical work up (i.e. clinical examination, electrophysiology and radiology) of patients suffering from CSM.

Volumetric MRI provides information on (macro-structural) general tissue loss (e.g. atrophy), whereas DTI provides information on fiber orientation and tissue structure and thus is sensitive to microstructural tissue properties. While decreased FA has been associated with both axonal count ${ }^{12}$ and myelin content ${ }^{13}$, increased RD and decreased $\mathrm{AD}$ have been associated with demyelination ${ }^{14}$ and axonal degeneration ${ }^{15}$, respectively. 

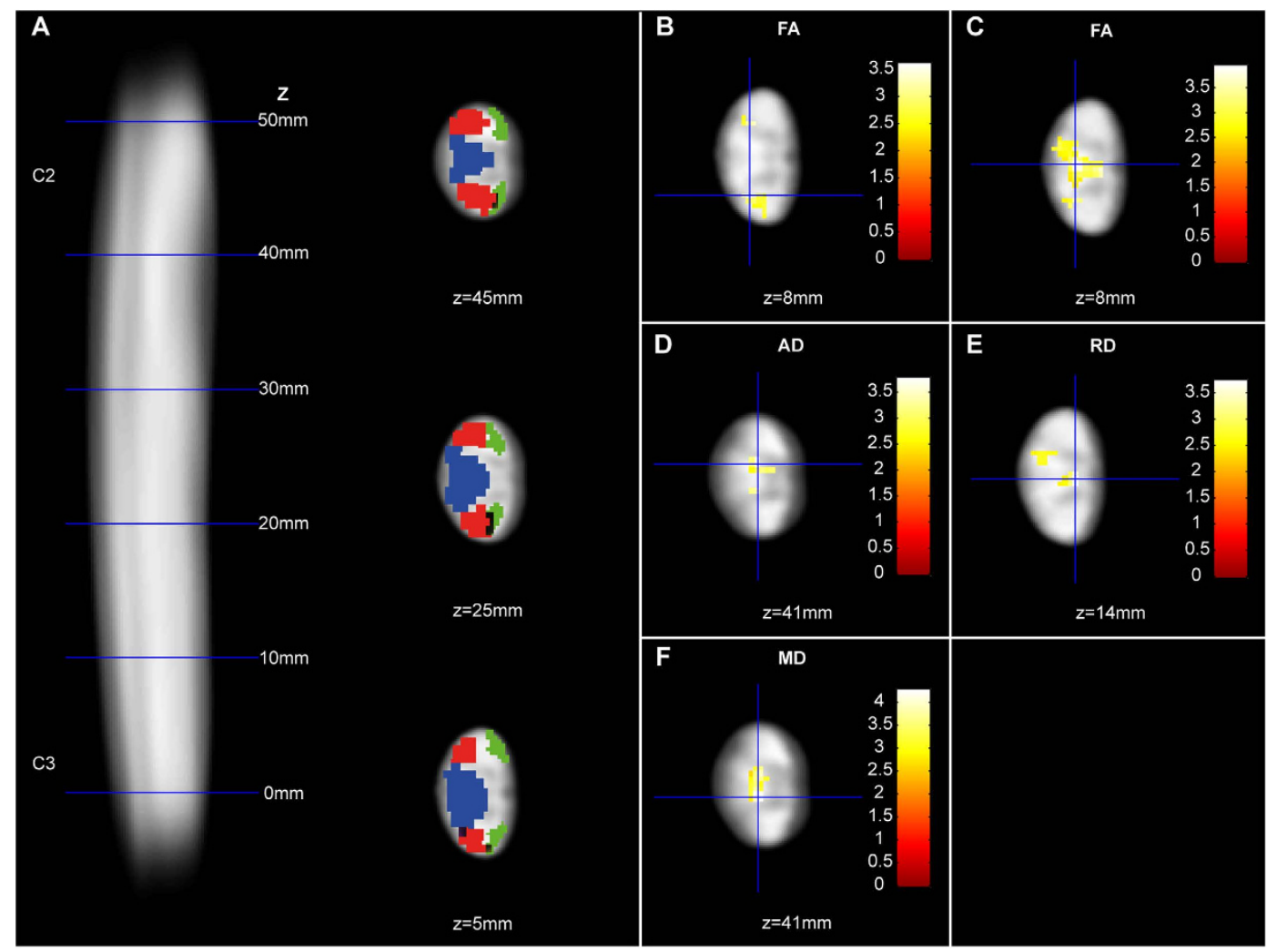

Figure 2. Tract-specific changes in microstructure above stenosis in patients with CSM compared to healthy controls. (A) Sagittal FA template of all subjects showing the cervical spinal cord at C2/C3 level and axial slices overlaid with regions of interest (lateral corticospinal tracts (CST) in red, posterior columns (PC) in blue, and spinothalamic tracts in green) for labeling of the anatomical position of the findings. (B-F) Overlay of statistical parametric maps ( $\mathrm{p}<0.01$ uncorrected, shown for descriptive purposes, masked by the lateral CST and $\mathrm{PC}$, respectively) revealing microstructural changes in patients compared to controls. In patients compared to controls, fractional anisotropy (FA) was reduced in the right lateral CST (B). In the PC, FA was reduced (C) and axial $(\mathbf{D})$, radial $(\mathbf{E})$, and mean diffusivity $(\mathbf{F})$ increased. Colour bars indicate t-values. Axial slices are shown and their position is indicated by the $\mathrm{z}$ coordinate.

This study found marked tissue loss (i.e. atrophy) of white and grey matter and tract-specific microstructural changes within major ascending and descending spinal pathways above the site of cord compression. The degree of atrophy above the site of stenosis was greater in the white matter $(-13.9 \%)$ and less strong in grey matter $(-7.2 \%)$. Changes in the spinal cord microvasculature ${ }^{3}$ and distribution of blood flow ${ }^{6,16}$, disruption of blood spinal cord barrier ${ }^{3}$, and neuroinflammation ${ }^{2,3}$ could make motoneurons above the compression site vulnerable to degeneration and apoptosis and be potential mechanisms for grey matter atrophy.

Moreover, we found tract-specific decreased FA within the lateral CST and PC, and increased RD and MD in the $\mathrm{PC}$ as markers of axonal degeneration and demyelination ${ }^{12-15,17,18}$ next to the reported white matter atrophy. These findings are coherent with other studies ${ }^{19-21}$. We found AD to be increased within the PC and these results are consistent with regards to the change of $\mathrm{AD}$ as shown in previous studies of $\mathrm{CSM}^{20,22,23}$. This might be due to elevated fiber tract density due to compression and loss of surrounding structure, as reported at the lesion site in patients with $\mathrm{CSM}^{24}$, but also caudal and rostral to the compression site ${ }^{25}$, that manifests over time. AD has been shown to behave bi-directionally over time in patients with optic neuritis and in experimental spinal contusion studies ${ }^{26,27}$. In the latter $\mathrm{AD}$ decreased rapidly in rostrocaudal direction, but increased over time at the lesion site $^{27}$. Nevertheless, in our study we report that the difference between patients with CSM and healthy controls in $\mathrm{RD}$ is nearly double as high as in $\mathrm{AD}$ which is suggestive that demyelination rather than axonal degeneration is occurring above the level of stenosis. It would be interesting to investigate our reported findings in other spinal cord pathologies at the same cervical level. Our conjoint analysis of macro- as well as microstructural changes are of interest because they suggest that a combination of pathological processes at the microstructural level including long distance retro- as well as anterograde fiber degeneration (i.e. axonal loss, demyelination) and potentially inflammation ${ }^{3}$ and to a lesser extent neuronal changes (i.e. apoptosis ${ }^{2,3}$ and anterior horn cell loss ${ }^{6,28}$ ) occur several segments above the level of stenosis.

The magnitude of remote changes in terms of atrophy and microstructural changes resembles those observed in traumatic spinal cord injury (SCI) ${ }^{18,29-33}$. Although based on two very different underlying aetiologies (abrupt onset in traumatic SCI, slowly developing symptoms in CSM), CSM and SCI clearly result in focal damage to the spinal cord with a combination of alpha-motoneuron damage (lesion of the central grey) as well as demyelination and axonal damage of long projecting spinal nerve fibre tracts (white matter damage) ${ }^{1,34}$. Herein we provide 


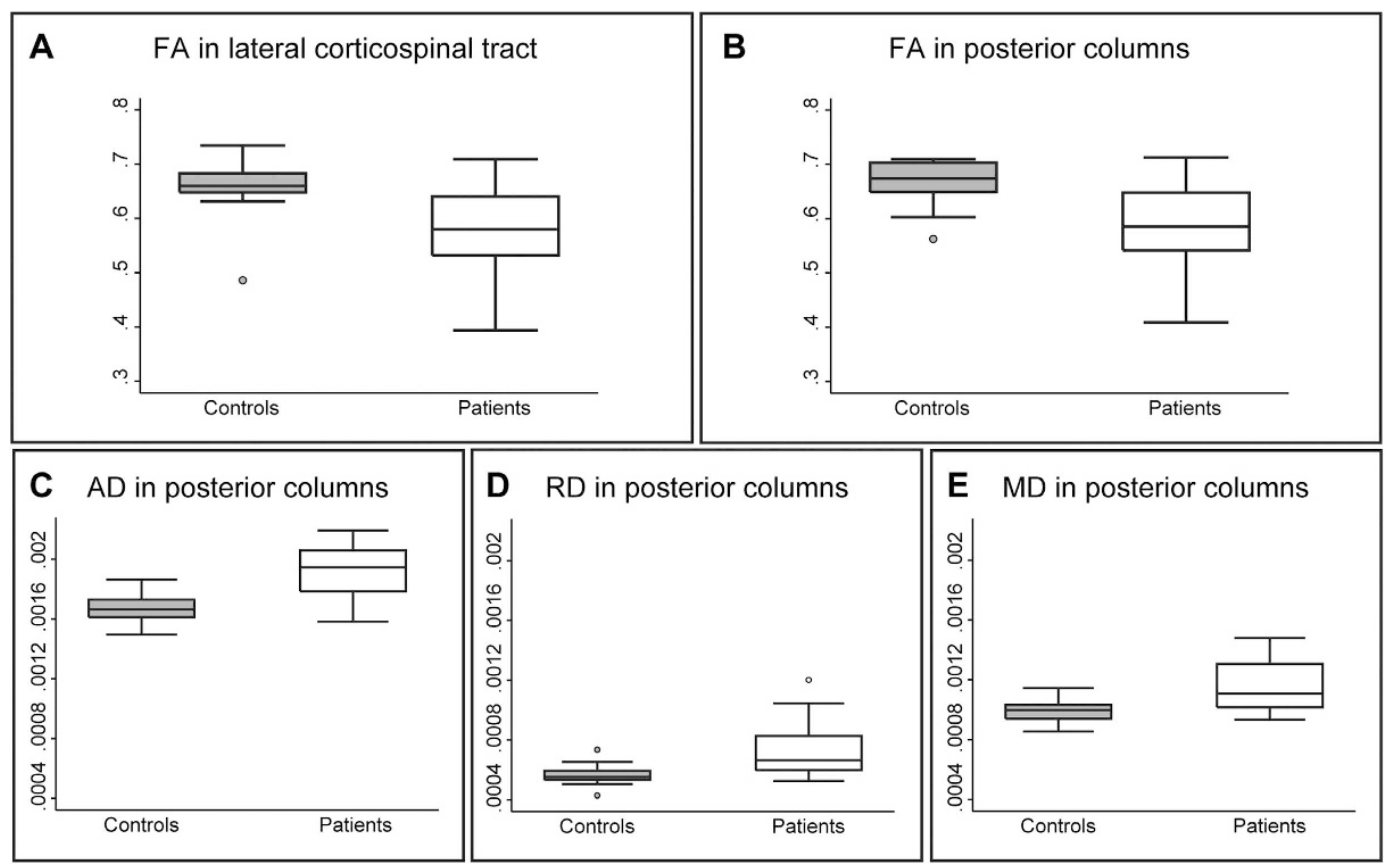

Figure 3. Quantification of tract-specific changes in microstructure above stenosis. In patients compared to controls, fractional anisotropy (FA) was reduced in the lateral CST (A) and PC (B), while AD (C), RD (D), and MD (E) were all increased in the PCs. Mean data were extracted from each significant cluster.

converging evidence that both aetiologies show remote cord pathology to a similar extent despite their striking difference in levels of disability. Given the marked degenerative changes above the site of stenosis, we anticipate atrophy and microstructural changes also to occur caudal to the site of stenosis contributing to the frequently seen gait disturbance ${ }^{1}$.

Associations between clinical outcomes and cord pathology at the level of cord damage have been reported previously ${ }^{11,24,35}$. Here we show that the structural changes above the site of stenosis carried within specific tracts are related to measures of functional independence (i.e. SCIM) within the lateral CST and sensory deficits (i.e. UELT) within the PC. The latter finding is striking as it suggests that greater structural perturbations, most probably corresponding to a combination of pathologic processes, contribute to sensory impairment. In contrast, despite signs of marked structural integrity changes of the lateral CST, most patients had normal or only mild pyramidal impairment. In other words, good clinical scores but marked structural changes may portend risk for a progressive functional decline.

We note the following considerations and limitations of this study. Firstly, the average age difference between gender matched patients and healthy controls was 7.6 years though this difference was not statistically significant. In all models, we included age as a regressor of no interest to reduce effects of age on our results. Secondly, the segmentation of the cervical cord into grey matter and posterior columns was performed manually, but the inter-, and intra-rater coefficients of variation for the cervical cord were similar to those published ${ }^{36}$. Finally, VBM-style analysis of spinal cord DTI is in its infancy ${ }^{7,9,10}$ and as with the early days of exploring brain DTI, there are limitations associated with this method. For example, spatial mis-registration can lead to false positive or false negative results and thus reduce the reliability of this emerging post-processing method for spinal cord DTI ${ }^{8}$. To reduce these, we performed an additional registration step to reduce residual mis-registrations that were not sufficiently removed by our proposed automated processing method. In future work, advanced spatial normalization methods such as Dartel ${ }^{37}$ could, if adopted to the spinal cord, help to improve registration quality and thus further advance VBM-style analysis of DTI in the spinal cord.

Another important issue of spinal cord DTI is the fact that instrumental and physiological noise artefacts are larger in the spinal cord than in the brain. This can reduce the reproducibility of spinal cord DTI metrics. To minimize artefacts, we applied sophisticated imaging and preprocessing methods. In particular, we reduced fold-over artefacts due to incomplete outer-volume suppression using $50 \%$ phase oversampling and by adjusting the amplitude of the saturation pulse manually for each subject. To minimize physiological noise artefacts, all patients were equipped with a stiff neck to reduce motion in the z-direction during scanning ${ }^{36}$. Further, we used a pulse-triggered sequence and optimized pre-processing techniques ${ }^{38}$ to mitigate the adverse effect of subject motion and other physiology-related artefacts (e.g. heart-beat or breathing). To assess the amount of variability due to residual artefacts, we tested the scan-rescan reliability of our data using a jackknife analysis as described in Mohammadi et al. (2013) (data analyses not shown). We found that the intra-subject variability (averaged across subjects) was about $\Delta$-FA $=0.02$, which is $3 \%$ when compared to the reported averaged FA values in the spinal cord $\left(0.02 / 0.66^{\star} 100=3 \%\right)$. This is smaller than the reported inter-subject variability of $\Delta$-FA $=0.05$ (reported in the Results section), which corresponds to $8 \%$ variability $\left(0.05 / 0.66^{\star} 100 \%=8 \%\right)$. Thus, we expect 


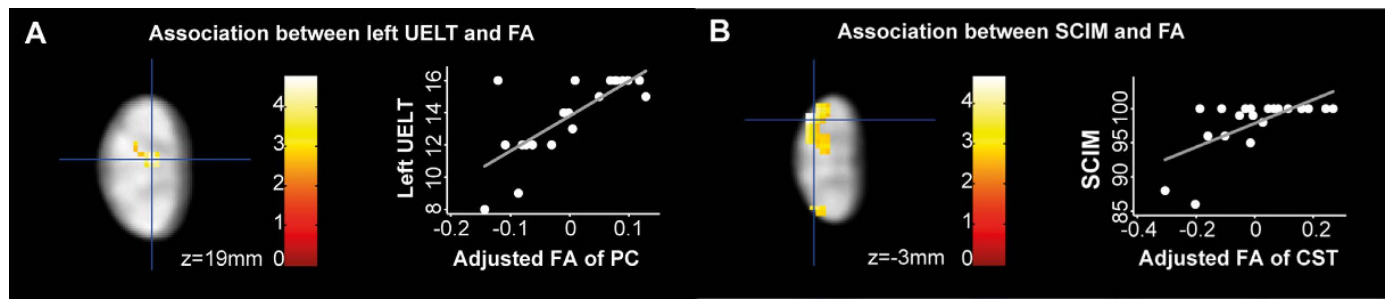

Figure 4. Associations between tract-specific microstructural readouts and clinical outcome above stenosis in patients with CSM. (A,B) Overlay of statistical parametric maps $(\mathrm{p}<0.01$ uncorrected, shown for descriptive purposes, masked by the lateral corticospinal tract (CST) and posterior columns (PC), respectively) revealing associations between DTI indices and clinical outcome (left panel) between cervical level C2 and C3 (see Fig. 2A for reference). Colour bars indicate t-values. The right panels illustrate the corresponding regression models extracted from the peak-voxel within the significant cluster. Diffusivity parameters of the correlations are mean centered and adjusted for age. In particularly, (A) lower fractional anisotropy (FA) in left PC was associated with worse left ISNCSCI upper extremity light-touch score (UELT) and (B) lower FA in the left lateral CST was associated with lower Spinal Cord Independence Measure (SCIM).

that the scan-rescan variability will not strongly alter the reported effects observed between controls and patients. However, we cannot exclude that the reported high inter-subject variability might be due to the fact that data quality varies between subjects, e.g. variation in motion artefacts between subjects.

To further improve the reproducibility of spinal cord DTI, optimizations at the level of the sequence (e.g. navigators that help discarding poor-quality scans as used in segmented $\mathrm{EPI}^{39}$ ) and post-processing techniques (e.g. denoising) as used in brain $\mathrm{DTI}^{40}$ should be considered.

\section{Conclusion}

Atrophy and tract-specific degeneration of the cervical cord can be detected by means of high-resolution MRI and expands far beyond focal cord damage as assessed by conventional MRI (e.g. MCC, MSCC, and signal change ratio). Importantly, these remote tract-specific changes are related to the clinical presentation and may be applicable as surrogate markers to assess treatment effects (in surgical and drug based interventions) complimentary to clinical outcome measures. Thus, MRI findings above and potentially below the site of stenosis could disclose subclinical sensory and motor impairments of upper and lower limb impairment and complement the routine clinical and electrophysiological assessment ${ }^{41}$ in individuals with CSM.

\section{Material and Methods}

Subjects and study design. Twenty patients (six women) (Table 1) referred for a neurological evaluation due to CSM with a mean age of $52.0 \pm 14.5$ (SD) years and 18 gender-matched controls (six women) with a mean age of $44.4 \pm 9.7$ (SD) years were consecutively recruited for this study between July 2012 and September 2014 in the outpatient clinic at the University Hospital Balgrist, Switzerland. The mean age between both groups was statistically not different (Mann-Whitney $\mathrm{U}$ test: $\mathrm{z}=-1.61, \mathrm{p}=0.1075)$. The participants fulfilled the following inclusion criteria: no other neurological or mental disorders affecting clinical outcome, age between 18 to 70 years, no MRI contradictions, and no pregnancy. The study protocols were in accordance with the Declaration of Helsinki and were approved by the local Ethics Committee of Zurich, the 'Kantonale Ethikkommission Zurich' (ref. number: EK-2012-0343). All participants provided written informed consent.

Clinical examination. All patients received a comprehensive clinical examination including the modified Japanese Orthopedic Association (mJOA) scale ${ }^{42}$ [max. 18 points], and additional outcome measures such as the ISNCSCI protocol ${ }^{43}$ for UEMS (e.g. pyramidal dysfunction) [max. 50 points], UELT (e.g. posterior column dysfunction) [max. 32 points], and UEPP (e.g. spinothalamic dysfunction) [max. 32 points], SCIM [max. 100 points] $)^{44}$, and the GRASSP protocol [max. 232 points] as ancillary outcome measure sensitive for upper extremities in $\mathrm{CSM}^{45}$ (sub-item for strength [max. 100 points], sensibility [max. 48 points], and prehension [max. 84 points $])^{46}$. Based on the mJOA, CSM severity was identified as mild $(\mathrm{mJOA} \geq 15)$, moderate $(\mathrm{mJOA}=12-14)$, or severe $(\mathrm{mJOA}<12)^{47}$.

Image acquisition. All participants were scanned on a 3T Skyra MRI scanner (Siemens Healthcare, Erlangen, Germany) equipped with a 16-channel radio-frequency (RF) receive head and neck coil and RF body transmit coil. All participants wore an MRI-compatible stiff neck (Laerdal Medicals, Stavanger, Norway) to minimize motion artefacts and were carefully positioned by the radiographers to acquire the data from the same position and to obtain high reproducibility between all participants.

A 2D sagittal T2-weighted turbo spin-echo sequence was used for anatomical assessment of cervical spinal cord covering the levels of stenosis. Following parameters were applied to acquire 20 slices within 2 minutes and 2 seconds: slice thickness $2.5 \mathrm{~mm}$ with $10 \%$ inter-slice gap, field of view (FOV) of $220 \times 220 \mathrm{~mm}^{2}$, matrix size of $384 \times 384$, time of repetition (TR) of $3760 \mathrm{~ms}$, time of echo (TE) of $87 \mathrm{~ms}$, flip angle $\alpha=160^{\circ}$, and readout bandwidth of $260 \mathrm{~Hz}$ per pixel.

A 3D high-resolution optimized $2^{*}$-weighted multi-echo sequence (multiple echo data image combination; MEDIC) ${ }^{48}$ was applied to acquire five high-resolution axial 3D volumes of the cervical cord above 
the stenosis at C2/C3 level. Each volume consisted of twenty partitions and was obtained with a resolution of $0.25 \times 0.25 \times 2.50 \mathrm{~mm}^{3}$ within 2 minutes and 8 seconds for each of the five volumes. Following parameters were applied: FOV of $162 \times 192 \mathrm{~mm}^{2}$, matrix size of $648 \times 768$, TR of $44 \mathrm{~ms}$, TE of $19 \mathrm{~ms}$, flip angle $\alpha=11^{\circ}$, and readout bandwidth of $260 \mathrm{~Hz}$ per pixel.

The acquisition of DTI data used a cardiac-gated monopolar sequence (based on finger pulse oximetry $)^{49}$ and the following parameters: 30 diffusion-weighted (DW) images $\left(b=500 \mathrm{~s} / \mathrm{mm}^{2}\right)$, six T2- weighted images without diffusion weighting $(\mathrm{b}=0), 5 \mathrm{~mm}$ slice thickness, with $10 \%$ inter-slice gap, 10 slices perpendicularly oriented to the spine, 5/8 Partial-Fourier Imaging in phase-encoding direction, phase oversampling 50\%, and a cardiac trigger delay of $200 \mathrm{~ms}, 176 \times 40$ acquisition matrix, FOV of $133 \times 30 \mathrm{~mm}^{2}, 0.8 \times 0.8 \mathrm{~mm}^{2}$ in-plane resolutions, TE of $73 \mathrm{~ms}$, and TR of $350 \mathrm{~ms}$. The gated data were acquired in blocks of two slices per cardiac cycle. The minimal time between successive triggers was $1800 \mathrm{~ms}$. Reduced field of view was achieved by outer-volume suppression ${ }^{49}$ using two spatial saturation pulses placed anterior and posterior to the spinal cord, along the phase-encoding direction ${ }^{50}$. To reduce fold-over artefacts due to insufficient outer volume suppression, the amplitude of the saturation pulse was adjusted for each subject individually. Each DTI dataset was acquired four times at the same $\mathrm{C} 2 / \mathrm{C} 3$ position in the cervical spinal cord, resulting in 144 images for each subject. Altogether, this resulted in a total acquisition time of about 6.2 minutes (as estimated by the sequence simulator), but could be longer depending on the participant's heart rate.

Processing of sagittal T2-weighted data of cervical spine. We used JIM 6.0 (Xynapse Systems, Aldwincle, UK) to calculate the maximum canal compromise (MCC) and maximum spinal cord compression (MSCC) using the midsagittal slice ${ }^{11,51}$. We calculated the signal change ratio from the region of hyperintensity or, if not applicable, from the level of greatest cord compression against an average reference on the spinal cord at $\mathrm{C} 7 / \mathrm{T} 1$ and $\mathrm{C} 2$ using the ratio proposed by Nouri et al. ${ }^{11}$.

Processing of high-resolution structural data above the level of stenosis. We used a symmetric diffeomorphic algorithm ${ }^{52}$, that is embedded in SPM12 (Wellcome Trust Centre for Neuroimaging, University College London, London, UK), to register the five 3D MEDIC volumes in each subject in order to account for non-rigid body motion effects and create an average volume for a better signal to noise ratio (SNR). Jim 6.0 was then used to merge the adjacent partitions to get 10 contiguous slices (to increase SNR) and to semi-automatically segment the cross-sectional cervical cord area using an active-surface model after setting a marker in the center of the cord in each of the 10 contiguous slices ${ }^{53}$. The GM and PC were extracted manually in each slice. WM was estimated by subtracting the GM from cervical cord area. The segmentation was performed by two independent investigators (PG and AT) and was reevaluated a second time with a gap of at least two weeks in between. Coefficient of variations (=standard deviation divided by mean) for cervical cord area, WM, GM, and PC were $0.6 \%, 1.9 \%, 6.9 \%$, and $3.4 \%$ for inter-rater and $0.9 \%, 1.3 \%, 4.4 \%$, and $1.4 \%$ for intra-rater variations, respectively.

Pre-processing and estimation of DTI data. The DTI data were interpolated to a higher in-plane resolution of $0.4 \times 0.4 \mathrm{~mm}^{2}$. Then, the data were corrected for motion and eddy current artefacts using a novel constrained $3 \mathrm{D}$-affine registration ${ }^{54}$ that corrected for selected rigid-body subject motion and linear eddy current distortion parameters (details on linear eddy currents can be found $\mathrm{in}^{55}$ ). We restricted the 3D-affine registration to correct only for in-plane $\mathrm{x}$ - and $\mathrm{y}$-translation (mainly caused by subject motion and eddy currents) as well as for in-plane scaling and in-plane shearing along the y-direction (both mainly caused by eddy currents). We did not correct for rotation and through-plane shearing effects, which were less pronounced and less robust to estimate. To correct for residual artefacts associated with subject motion and physiological noise ${ }^{38}$, we used robust fitting as implemented in the ACID toolbox (www.diffusiontools.com). Combining eddy current and motion correction with the robust tensor fitting approach has been shown to be particularly efficient to reduce intra-scan variability of DTI metrics ${ }^{56}$.

Post-processing and spatial normalization of DTI index maps. First, the in-plane field-of-view was chopped to $24 \times 24 \mathrm{~mm}^{2}$ for each DTI dataset to exclude non-spine tissue. Then, the following pathological relevant DTI index maps ${ }^{9,13,29}$ were calculated: $F A, R D, A D$, and $M D$. These DTI index maps were spatial normalized into the space of the AMU white and grey matter probabilistic tracts embedded within the MNI-Poly-AMU template $^{7}$ using the FA voxel-based statistics (FA-VBS) toolbox ${ }^{8}$ with refined spatial normalization parameters, which specifically accounted for the anatomy of the spinal cord, i.e. the degree of freedom of the spatial transformation was reduced along the head-foot direction. To use optimal and complementary contrast information, we used the MD and FA contrast to drive the spatial normalization. Then, we performed additional manual slice-by-slice registration (shift and scaling along the phase-encoding direction) to refine to accuracy of the registration. Finally, all DTI index maps were smoothed using a Gaussian kernel with $0.5 \times 0.5 \times 5 \mathrm{~mm}^{3}$ with full-width at half maximum (FWHM).

Statistical analysis. Stata 13 (StataCorp LP, Texas, USA) was used for statistical analysis of clinical and morphometric data. We used Mann-Whitney U tests to investigate age differences between patients and controls. We used ANCOVA with age as covariate of no interest to assess morphometric differences between patients and controls in cross-sectional cervical cord area, GM, WM, and PC. Linear regression models were used to determine associations between bilateral clinical outcome measures for mJOA, UELT, UEPP, GRASSP sensibility, strength, and prehension, SCIM, MSCC, MCC, and signal change ratio and cross-sectional cervical cord area and between UELT and GRASSP sensibility and PC. Bilateral outcome measures were used because there is not landmark to segment left and right cross-sectional cervical cord measures separately. Age was included and treated as covariate 
of no interest to adjust for possible confounds of age in every statistical test. The level of significance was defined as $\alpha=0.050$.

SPM12 was used for voxel-based statistics of the different DTI indices. To this end, we investigated the averaged DTI indices across the cross-section of the cervical cord (C2/C3) and verified the tract-specific location using the white matter spinal cord atlas that is embedded in the Spinal Cord Toolbox ${ }^{57}$ for the PC, lateral CST, and spinothalamic tracts. We used ANCOVA to investigate cross-sectional differences in DTI indices (e.g. FA, AD, $\mathrm{RD}$, and $\mathrm{MD}$ ) between patients and controls and adjusted for age within the groups.

Linear regression models were constructed for associations between unilateral clinical outcome measures for UELT, UEPP, GRASSP sensibility, strength, and prehension and DTI indices (except for mJOA, SCIM, MSCC, MCC, and signal change ratio as purely bilateral outcome measures). Age was included as a covariate of no interest in all linear regression models to adjust for possible confounds of age. All statistical parametric maps were initially thresholded with a cluster defining threshold of uncorrected $\mathrm{p}<0.01$. Clusters surpassing a cluster threshold of $\mathrm{p}=0.05$, corrected for family-wise error based on Gaussian Random Field theory, are reported ${ }^{58}$.

\section{References}

1. Kalsi-Ryan, S., Karadimas, S. K. \& Fehlings, M. G. Cervical spondylotic myelopathy: the clinical phenomenon and the current pathobiology of an increasingly prevalent and devastating disorder. Neuroscientist 19, 409-421 (2013).

2. Yu, W. R., Liu, T., Kiehl, T.-R. \& Fehlings, M. G. Human neuropathological and animal model evidence supporting a role for Fasmediated apoptosis and inflammation in cervical spondylotic myelopathy. Brain 134, 1277-1292 (2011).

3. Karadimas, S. K. et al. A novel experimental model of cervical spondylotic myelopathy (CSM) to facilitate translational research. Neurobiol. Dis. 54, 43-58 (2013).

4. Holly, L. T., Moftakhar, P., Khoo, L. T., Shamie, A. N. \& Wang, J. C. Surgical outcomes of elderly patients with cervical spondylotic myelopathy. Surg. Neurol. 69, 233-240 (2008).

5. Moon, E. S., Karadimas, S. K., Yu, W. R., Austin, J. W. \& Fehlings, M. G. Riluzole attenuates neuropathic pain and enhances functional recovery in a rodent model of cervical spondylotic myelopathy. Neurobiol. Dis. 62, 394-406 (2014).

6. Karadimas, S. K. et al. Riluzole blocks perioperative ischemia-reperfusion injury and enhances postdecompression outcomes in cervical spondylotic myelopathy. Sci. Transl. Med. 7, 316ra194 (2015).

7. Fonov, V. S. et al. Framework for integrated MRI average of the spinal cord white and gray matter: The MNI-Poly-AMU template. Neuroimage 102 Pt 2, 817-827 (2014).

8. Mohammadi, S. et al. The influence of spatial registration on detection of cerebral asymmetries using voxel-based statistics of fractional anisotropy images and TBSS. PLos One 7, e36851 (2012).

9. Toosy, A. T. et al. Voxel-based cervical spinal cord mapping of diffusion abnormalities in MS-related myelitis. Neurology 83, $1321-1325$ (2014).

10. Naismith, R. T. et al. Spinal cord tract diffusion tensor imaging reveals disability substrate in demyelinating disease. Neurology $\mathbf{8 0}$, 2201-2209 (2013).

11. Nouri, A. et al. Role of Magnetic Resonance Imaging in Predicting Surgical Outcome in Patients With Cervical Spondylotic Myelopathy. Spine (Phila. Pa. 1976). 40, 171-178 (2015).

12. Gouw, A. A. et al. Heterogeneity of white matter hyperintensities in Alzheimer's disease: post-mortem quantitative MRI and neuropathology. Brain 131, 3286-3298 (2008).

13. Schmierer, K. et al. Diffusion tensor imaging of post mortem multiple sclerosis brain. Neuroimage 35, 467-477 (2007).

14. Klawiter, E. C. et al. Radial diffusivity predicts demyelination in ex vivo multiple sclerosis spinal cords. Neuroimage 55, 1454-1460 (2011).

15. Zhang, J. et al. Diffusion tensor magnetic resonance imaging of Wallerian degeneration in rat spinal cord after dorsal root axotomy. J. Neurosci. 29, 3160-3171 (2009).

16. Kurokawa, R., Murata, H., Ogino, M., Ueki, K. \& Kim, P. Altered blood flow distribution in the rat spinal cord under chronic compression. Spine (Phila. Pa. 1976). 36, 1006-1009 (2011).

17. Budde, M. D., Xie, M., Cross, A. H. \& Song, S.-K. Axial diffusivity is the primary correlate of axonal injury in the experimental autoimmune encephalomyelitis spinal cord: a quantitative pixelwise analysis. J. Neurosci. 29, 2805-2813 (2009).

18. Cohen-Adad, J. et al. Demyelination and degeneration in the injured human spinal cord detected with diffusion and magnetization transfer MRI. Neuroimage 55, 1024-1033 (2011).

19. Zhou, F. Q. et al. Intrinsic Functional Plasticity of the Sensory-Motor Network in Patients with Cervical Spondylotic Myelopathy. Sci. Rep. 5, 9975 (2015).

20. Cui, J.-L. et al. Quantitative assessment of column-specific degeneration in cervical spondylotic myelopathy based on diffusion tensor tractography. Eur. Spine J. 24, 41-47 (2014).

21. Wen, C. Y. et al. Is diffusion anisotropy a biomarker for disease severity and surgical prognosis of cervical spondylotic myelopathy? Radiology 270, 197-204 (2014).

22. Wen, C. Y., Cui, J. L., Mak, K. C., Luk, K. D. K. \& Hu, Y. Diffusion tensor imaging of somatosensory tract in cervical spondylotic myelopathy and its link with electrophysiological evaluation. Spine J. 14, 1493-1500 (2014).

23. Rajasekaran, S. et al. The Assessment of Neuronal Status in Normal and Cervical Spondylotic Myelopathy Using Diffusion Tensor Imaging. Spine (Phila. Pa. 1976). 39, 1183-1189 (2014).

24. Ellingson, B. M., Salamon, N., Woodworth, D. C. \& Holly, L. T. Correlation between degree of subvoxel spinal cord compression measured with super-resolution tract density imaging and neurological impairment in cervical spondylotic myelopathy. $J$. Neurosurg. Spine 22, 631-638 (2015).

25. Yu, W. R. et al. Molecular mechanisms of spinal cord dysfunction and cell death in the spinal hyperostotic mouse: Implications for the pathophysiology of human cervical spondylotic myelopathy. Neurobiol. Dis. 33, 149-163 (2009).

26. Naismith, R. T. et al. Disability in optic neuritis correlates with diffusion tensor-derived directional diffusivities. Neurology 72 , 589-594 (2009).

27. Ellingson, B. M., Kurpad, S. N., Li, S.-J. \& Schmit, B. D. In vivo diffusion tensor imaging of the rat spinal cord at 9.4T. J. Magn. Reson. Imaging 27, 634-642 (2008).

28. Fehlings, M. G. \& Skaf, G. A review of the pathophysiology of cervical spondylotic myelopathy with insights for potential novel mechanisms drawn from traumatic spinal cord injury. Spine (Phila. Pa. 1976). 23, 2730-2737 (1998).

29. Freund, P. et al. Axonal integrity predicts cortical reorganisation following cervical injury. J. Neurol. Neurosurg. Psychiatry 83, 629-637 (2012).

30. Freund, P. et al. Disability, atrophy and cortical reorganization following spinal cord injury. Brain 134, 1610-1622 (2011).

31. Freund, P. et al. MRI investigation of the sensorimotor cortex and the corticospinal tract after acute spinal cord injury: a prospective longitudinal study. Lancet Neurol. 12, 873-881 (2013).

32. Lundell, H. et al. Independent spinal cord atrophy measures correlate to motor and sensory deficits in individuals with spinal cord injury. Spinal Cord 49, 70-75 (2011). 
33. Grabher, P. et al. Tracking sensory system atrophy and outcome prediction in spinal cord injury. Ann. Neurol. 78, 751-761 (2015).

34. Buss, A. et al. Sequential loss of myelin proteins during Wallerian degeneration in the human spinal cord. Brain 128, 356-364 (2005).

35. Arvin, B. et al. Preoperative magnetic resonance imaging is associated with baseline neurological status and can predict postoperative recovery in patients with cervical spondylotic myelopathy. Spine (Phila. Pa. 1976). 38, 1170-1176 (2013).

36. Yiannakas, M. C. et al. Feasibility of grey matter and white matter segmentation of the upper cervical cord in vivo: a pilot study with application to magnetisation transfer measurements. Neuroimage 63, 1054-1059 (2012).

37. Ashburner, J. A fast diffeomorphic image registration algorithm. Neuroimage 38, 95-113 (2007).

38. Mohammadi, S., Hutton, C., Nagy, Z., Josephs, O. \& Weiskopf, N. Retrospective correction of physiological noise in DTI using an extended tensor model and peripheral measurements. Magn. Reson. Med. 70, 358-369 (2013).

39. Porter, D. A. \& Heidemann, R. M. High resolution diffusion-weighted imaging using readout-segmented echo-planar imaging, parallel imaging and a two-dimensional navigator-based reacquisition. Magn. Reson. Med. 62, 468-475 (2009).

40. Tabelow, K., Mohammadi, S., Weiskopf, N. \& Polzehl, J. POAS4SPM: a toolbox for SPM to denoise diffusion MRI data. Neuroinformatics 13, 19-29 (2015).

41. Ulrich, A., Haefeli, J., Blum, J., Min, K. \& Curt, A. Improved diagnosis of spinal cord disorders with contact heat evoked potentials. Neurology 80, 1393-1399 (2013).

42. Benzel, E. C., Lancon, J., Kesterson, L. \& Hadden, T. Cervical laminectomy and dentate ligament section for cervical spondylotic myelopathy. Journal of spinal disorders 4, 286-295 (1991).

43. Kirshblum, S. C. et al. Reference for the 2011 revision of the International Standards for Neurological Classification of Spinal Cord Injury. J. Spinal Cord Med. 34, 547-554 (2011).

44. Anderson, K. et al. Functional Recovery Measures for Spinal Cord Injury: An Evedence-Based Review for Clinical Practice and Research. J. Spinal Cord Med. 31, 133-144 (2008).

45. Kalsi-Ryan, S. et al. Ancillary outcome measures for assessment of individuals with cervical spondylotic myelopathy. Spine (Phila. Pa. 1976). 38, S111-S122 (2013).

46. Kalsi-Ryan, S., Curt, A., Verrier, M. C. \& Fehlings, M. G. Development of the Graded Redefined Assessment of Strength, Sensibility and Prehension (GRASSP): reviewing measurement specific to the upper limb in tetraplegia. J. Neurosurg. Spine 17, 65-76 (2012).

47. Fehlings, M. G. et al. Efficacy and Safety of Surgical Decompression in Patients with Cervical Spondylotic Myelopathy. J. Bone Jt. Surg. 95, 1651-1658 (2013).

48. Schmid, M. R. et al. Imaging of patellar cartilage with a 2D multiple-echo data image combination sequence. AJR Am J Roentgenol 184, 1744-1748 (2005).

49. Morelli, J. N. et al. Evaluation of a modified Stejskal-Tanner diffusion encoding scheme, permitting a marked reduction in TE, in diffusion-weighted imaging of stroke patients at 3 T. Invest. Radiol. 45, 29-35 (2010).

50. Heidemann, R. M. et al. High resolution single-shot diffusion-weighted imaging with a combination of zoomed EPI and parallel imaging. in Proceedings of the 17th Annual Meeting of ISMRM, Honolulu, USA, p. 2736 (2009).

51. Fehlings, M. G. et al. The optimal radiologic method for assessing spinal canal compromise and cord compression in patients with cervical spinal cord injury. Part II: Results of a multicenter study. Spine (Phila. Pa. 1976). 24, 605-613 (1999).

52. Ashburner, J. \& Ridgway, G. R. Symmetric diffeomorphic modeling of longitudinal structural MRI. Front. Neurosci. 6, 1-19 (2013).

53. Horsfield, M. a et al. Rapid semi-automatic segmentation of the spinal cord from magnetic resonance images: application in multiple sclerosis. Neuroimage 50, 446-455 (2010).

54. Mohammadi, S. et al. High-resolution diffusion kurtosis imaging at 3T enabled by advanced post-processing. Front. Neurosci. 8, 1-14 (2015).

55. Mohammadi, S., Möller, H. E., Kugel, H., Müller, D. K. \& Deppe, M. Correcting eddy current and motion effects by affine wholebrain registrations: evaluation of three-dimensional distortions and comparison with slicewise correction. Magn. Reson. Med. 64, 1047-1056 (2010).

56. Mohammadi, S., Freund, P., Feiweier, T., Curt, A. \& Weiskopf, N. The impact of post-processing on spinal cord diffusion tensor imaging. Neuroimage 70, 377-385 (2013).

57. Cohen-Adad, J. et al. Spinal Cord Toolbox: an open-source framework for processing spinal cord MRI data. in Proceedings of the 20th Annual Meeting of OHBM, Hamburg, Germany, p. 3633 (2014).

58. Friston, K. J., Worsley, K. J., Frackowiak, R. S., Mazziotta, J. C. \& Evans, a. C. Assessing the significance of focal activations using their spatial extent. Hum. Brain Mapp. 1, 210-20 (1994).

\section{Acknowledgements}

We would like to thank all participants for spending their valuable time, the staff of the Department of Radiology and Neurology at the University Hospital Balgrist, Janosch Rienert for analysis of conventional MRI measures, and all other colleagues involved in this study. This study was funded by the Clinical Research Priority Program

"NeuroRehab" of the University of Zurich and the International Foundation for Research in Paraplegia . The Wellcome Trust Centre for Neuroimaging is supported by core funding from the Wellcome Trust 091593/Z/10/Z.

\section{Author Contributions}

The study was designed by S.M., R.S., N.W., A.J.T., A.C. and P.F. The data were acquired by P.G., A.T., S.F., R.S., A.C. and P.F. The analysis was performed by P.G., S.M., G.D., N.W. and P.F. P.G., S.M., N.W., A.J.T., A.C. and P.F. wrote the manuscript. All authors reviewed the paper. P.F. had full access to all the data in the study and takes responsibility for the integrity of the data and the accuracy of the data analysis.

\section{Additional Information}

\section{Supplementary information accompanies this paper at http://www.nature.com/srep}

Competing financial interests: Yes there is potential competing financial interest. P.G., S.M., A.T., S.F., G.D., R.S., A.C. and P.F. report no competing financial interests. Prof. Nikolaus Weiskopf reports that the Wellcome Trust Centre for Neuroimaging has an institutional research agreement with and receives support from Siemens Healthcare. Prof. Alan J. Thompson has received honoraria and support for travel for consultancy from Biogen Idec, MedDay, Eisai, and Novartis, and for teaching from Teva, Novartis, and EXCEMED. He receives an honorarium as editor-in-chief of Multiple Sclerosis Journal.

How to cite this article: Grabher, P. et al. Voxel-based analysis of grey and white matter degeneration in cervical spondylotic myelopathy. Sci. Rep. 6, 24636; doi: 10.1038/srep24636 (2016). 
(c) (i) This work is licensed under a Creative Commons Attribution 4.0 International License. The images or other third party material in this article are included in the article's Creative Commons license, unless indicated otherwise in the credit line; if the material is not included under the Creative Commons license, users will need to obtain permission from the license holder to reproduce the material. To view a copy of this license, visit http://creativecommons.org/licenses/by/4.0/ 